\title{
The Bioequivalence of Rasagiline Tablets in Chinese Healthy Subjects Under Fasting and Fed Conditions
}

\author{
yinjuan $\mathrm{li}^{1}, \mathrm{Lu} \mathrm{Qi}{ }^{1}$, ying $\mathrm{Liu}^{1}$, Rongxia $\mathrm{Fan}^{1}$, Yongrui $\mathrm{Tu}^{2}$, Yongqiang $\mathrm{Sun}^{3}$, Juxiang \\ Wang $^{3}$, Qi Qi ${ }^{4}$, Xiaohui Feng ${ }^{4}$, Da Zhou ${ }^{5}$, and Xinghe Wang ${ }^{1}$ \\ ${ }^{1}$ Capital Medical University Affiliated Beijing Shijitan Hospital \\ ${ }^{2}$ 2. Changzhou Siyao Pharmaceuticals Co.,Ltd \\ ${ }^{3}$ 2. Changzhou Siyao Pharmaceuticals Co.,Ltd \\ ${ }^{4}$. Changzhou Siyao Pharmaceuticals Co \\ 52.Changzhou Siyao Pharmaceuticals Co.
}

April 28, 2020

\begin{abstract}
Objective This study aims to evaluate the bioequivalence of 2 formulations of rasagiline tablet (1mg) in Chinese healthy subjects. Methods An open, randomized, single-dose, double-cycle, two-sequence, self-crossover pharmacokinetic study in healthy Chinese subjects under fasting and high-fat postprandial conditions was performed. A total of 108 healthy subjects (36 in the fasting group and 72 in the postprandial group) were recruited. In each of the two study periods under both conditions, subjects received a single oral dose of $1 \mathrm{mg}$ test or a reference rasagiline (1 $\mathrm{mg}$ each). There was a 3-day washout period. Blood samples were obtained up to 10 hours post-intake. Several pharmacokinetic parameters were estimated based on the concentrations of rasagiline measured in plasma by means of LC-MS/MS. Results The geometric mean ratio (90\% CI) of the test drug versus reference drug for rasagiline was $94.16 \%$ to $105.35 \%$ for AUC0-t under fasting conditions and $99.88 \%$ to $107.07 \%$ under postprandial conditions. The AUC0-[?]s were $93.55 \%$ to $105.01 \%$ and $99.59 \%$ to $107.05 \%$ under fasting and postprandial conditions, respectively. The Cmax values were $88.26 \%$ to $108.46 \%$ and $89.54 \%$ to $118.23 \%$ under two conditions, respectively. The $90 \%$ CIs for test/reference AUC ratio and Cmax ratio were within the acceptable range (0.80-1.25) for BE. There were no serious adverse events (AEs) encountered during this BE study. Conclusion Bioequivalence between the test and the reference products was established in both fasting and postprandial conditions. The two types of rasagiline showed good tolerability and a similar safety profile.
\end{abstract}

\begin{abstract}
:
Objective This study aims to evaluate the bioequivalence of 2 formulations of rasagiline tablet (1mg) in Chinese healthy subjects.Methods An open, randomized, single-dose, double-cycle, two-sequence, self-crossover pharmacokinetic study in healthy Chinese subjects under fasting and high-fat postprandial conditions was performed. A total of 108 healthy subjects (36 in the fasting group and 72 in the postprandial group) were recruited. In each of the two study periods under both conditions, subjects received a single oral dose of $1 \mathrm{mg}$ test or a reference rasagiline (1 mg each). There was a 3-day washout period. Blood samples were obtained up to 10 hours post-intake. Several pharmacokinetic parameters were estimated based on the concentrations of rasagiline measured in plasma by means of LC-MS/MS. Results The geometric mean ratio (90\% CI) of the test drug versus reference drug for rasagiline was $94.16 \%$ to $105.35 \%$ for $\mathrm{AUC}_{0-\mathrm{t}}$ under fasting conditions and $99.88 \%$ to $107.07 \%$ under postprandial conditions. The $\mathrm{AUC}_{0-[?]} \mathrm{S}$ were $93.55 \%$ to $105.01 \%$ and $99.59 \%$ to $107.05 \%$ under fasting and postprandial conditions, respectively. The $\mathrm{C}_{\max }$ values were $88.26 \%$ to $108.46 \%$ and $89.54 \%$ to $118.23 \%$ under two conditions, respectively. The $90 \%$ CIs for test/reference AUC ratio and
\end{abstract}


$\mathrm{C}_{\max }$ ratio were within the acceptable range (0.80-1.25) for BE. There were no serious adverse events (AEs) encountered during this BE study. ConclusionBioequivalence between the test and the reference products was established in both fasting and postprandial conditions. The two types of rasagiline showed good tolerability and a similar safety profile.

Key words: bioequivalence, rasagiline, monoamine oxidase type B inhibitor, pharmacokinetic

Parkinson's disease (PD) is a progressively disabling neurodegenerative disorder caused by the loss of dopaminergic neurons in the substantia nigra pars compacta. The clinical symptoms of PD patients include bradykinesia, muscle rigidity, resting tremor, and postural instability, which lead to gait disturbances and falls ${ }^{[1,2]}$. Second only to Alzheimer's disease (AD), PD is among the most common neurodegenerative diseases in the world, which affects around $0.3 \%$ of the general population and increases to $1-3 \%$ of people above 65 years of age. The number of people who suffer from PD is predicted to climb from 8.7 to 9.3 million by $2030^{[3]}$. A more recent meta-analysis estimated the overall prevalence and annual incidence on PD in China to be $16-440$ per 100,000 and $1.5-8.7$ per 100,000 people, respectively ${ }^{[4]}$. Currently, PD affects millions of people globally and has severe social and economic impacts.

Therapeutic agents to control the symptoms of PD include levodopa (a precursor in dopamine synthesis), dopamine agonists and monoamine oxidase type $\mathrm{B}$ (MAO-B) inhibitor, the major enzyme responsible for the oxidative metabolism of dopamine in the human brain. The first (MAO-B) inhibitor was selegiline, with a major drawback of metabolism to (-)-amphetamine and (-)-methamphetamine. These metabolites play an "anti-tyramine" role by inhibiting the uptake of dopamine by neurons, resulting in neurotoxicity and cardiovascular adverse effects ${ }^{[5-8]}$. Rasagiline mesylate is a potent, highly selective, and irreversible secondgeneration MAO-B inhibitor and has neuroprotective activity ${ }^{[8-9]}$. Its major metabolite is $1(\mathrm{R})$-aminoindan differentiated by distinctly non-amphetamine structural features ${ }^{[7]}$. Rasagiline mesylate (AZILECTß), developed jointly by Israel's Teva and Denmark's Lundbeck, was first approved by the European Medicines Agency (EMA) in February 2005 and approved by the United States FDA in 2006 as monotherapy in PD patients not treated with levodopa and as adjunct therapy to levodopa in levodopa-treated patients. In June 2017, rasagiline was approved by CFDA to be listed in China and is currently used by PD patients in over 50 countries taking doses ranging between $0.5 \mathrm{mg}$ and $1 \mathrm{mg}$ daily.

According to recent surveys conducted with healthy individuals and PD patients in China and Japan, rasagiline can safely and effectively treat Parkinson's disease symptoms by blocking the decomposition of the neurotransmitter dopamine, with good drug tolerance and long-lasting effects ${ }^{[10-13]}$. As a whole, it is a novel, safe and effective drug to manage Parkingson's disease.

From 2006 to 2016, there were only two MAO-B inhibitors (rasagiline and safinamide) successfuly been commercialized. Consequently, PD patients have limited choices of these drugs ${ }^{[6]}$. Although antiparkinsonian medications are not considered to be the most expensive pharmacological agents, lifelong treatment and often complex drug regimens impose a high economic burden on both patients and the healthcare system ${ }^{[14]}$. Accordingly, the development and application of generic drugs as substitutes for the branded ones is a clear and economic choice.

Rasagiline mesylate tablets produced by Changzhou Siyao Pharmaceuticals Co., Ltd. is the first generic drug of its type in China. Taking a test preparation and using AZILECT ${ }^{\circledR}$ as reference, this study investigates the pharmacokinetic $(\mathrm{PK})$ properties of rasagiline mesylate in healthy Chinese volunteers with single-dose administration under fasting or postprandial conditions and to assess the BE of the 2 rasagiline mesylate tablets. After the administration of a single $1 \mathrm{mg}$ oral dose on healthy Chinese volunteers, we applied a 2 -sequence and 2-period crossover $(2 \times 2)$ study design. This study was registered and approved by the China Food and Drug Administration.

\section{Materials and Methods}

This study was conducted in full accordance with the Declaration of Helsinki and Good Clinical Practice guidelines and applicable national and local laws and regulations. This study has been registered in Chine- 
se Clinical Trial Registry (ChiCTR) as ChiCTR1800017978 and Drug Trial Registration and Information Publication Platform (chinaDrugtrials.org.cn) as CTR20181466.

This study was performed in Phase I Clinical Trial Center, Beijing Shijitan Hospital affiliated with Capital Medical University ,Beijing, China, approved by the Independent Ethics Committee of participating institutions. All study participants provided signed informed consent and had the right to withdraw their consent at any time, without giving reason and without detriment.

\section{Study Design and Drug Administration}

A randomized, open, single-dose, double-cycle, two-sequence, crossover PK-BE study was designed to be conducted in healthy Chinese subjects under fasting and postprandial conditions. A washout period of 3 days was observed before crossing over to prevent the carry-over effect. 108 healthy Chinese volunteers, both male and female, were enrolled in this study, which was separated into two groups, 36 subjects for the fasting group and 72 subjects for the postprandial group. In each group, subjects were randomized into 2 treatment sequence groups: T-R or R-T (where $\mathrm{T}$ refers to the test tablet, and $\mathrm{R}$ refers to the reference tablet). The order of receiving the test and reference products for each subject during each study period was determined according to a randomization schedule generated by SAS 9.4 software by a biostatistician.

In the fasting group, each subject randomly received a single dose of the $\mathrm{T}$ or $\mathrm{R}$ tablet of $1 \mathrm{mg}$ rasagiline after overnight fasting for 10 hours. In the postprandial group, each subject received a single dose of the $\mathrm{T}$ or $\mathrm{R}$ tablet of $1 \mathrm{mg}$ rasagiline with a high-fat and high-calorie breakfast (total energy 1000 kilocalories). The study drug was administered with $240 \mathrm{~mL}$ of water. Additional water intake was forbidden 1 hour before and after administration.

Study Population

Healthy Chinese subjects age 18 and over, with body mass index (BMI) between 19.0 and $28.0 \mathrm{~kg} / \mathrm{m}^{2}$ were eligible for recruitment. Subjects needed to be in good health as determined by medical history, 12lead ECG, vital signs, physical examination and laboratory tests (hematology, blood biochemistry, blood pregnancy test, urinalysis, virological screening, alcohol breath test and other drugs of abuse) and nonsmoking status. Subjects were not allowed to take any medications or supplements throughout the study. To avoid hypertensive crisis, a diet rich in tyramine such as wine and cheese, was prohibited. Eligible subjects did not have concomitant conditions or treatments that could have interfered with the study or interpretation of its results. Women of childbearing age were eligible if they had a negative serum pregnancy test and were willing to use consistent and acceptable contraception for the duration of the study. Male subjects also agreed to use acceptable contraception during the study and for 6 months after the study administration. Enrolled subjects should not have participated in another clinical trial in the last 3 months.

\section{Study Drugs}

The reference tablet was rasagiline mesylate $\left(1 \mathrm{mg}\right.$ AZILECT ${ }^{\circledR}$, Lot No.R81689, expiration date September 2019) produced by Teva Pharmaceutical Industries Ltd. The test tablet was rasagiline mesylate (1mg, Lot No.20170214, expiration date February 2020) manufactured by Changzhou Siyao Pharmaceuticals Co.,Ltd., Jiangsu, China.

\section{Blood Sampling and Medical Supervision}

Pharmacokinetics Blood samples (4mL each) for pharmacokinetic analysis of rasagiline were collected 1 hour pre-dose and at 5, 10, 20,30, and 45 minutes, and 1.0, 1.33, 1.67, 2.0, 2.5, 3.0, 4.0, 6.0, 8.0, 10.0 hours postdose in postprandial group. While in the fasting group, blood samples were collected at the following time points: 1 hour pre-dose and at 5, 10, 15, 20, 30 and 45 minutes, and 1.0, 1.25, 2.0, 3.0, 4.0, 6.0, 8.0, 10.0 hours.

Subjects were confined to the study center for continuous medical supervision until all PK blood samples were collected the next day after the second period of administration. Safety assessments included physical 
examination, vital signs, ECG recording, adverse event (AE) reporting, clinical laboratory measures and concomitant medication reporting.

Assays of rasagiline

Blood samples were centrifuged at $2500 \mathrm{~g}$ a t $2-8^{\circ} \mathrm{C}$ for 10 minutes to obtain plasma samples. The plasma samples were stored at $-80^{\circ} \mathrm{C}$ which met the conditions for storage of stable samples in the validation of methodology. Plasma concentrations of rasagiline were analyzed using a validated liquid chromatographymass spectrometry method (LC-MS/MS). The linear range was 20.0 to $20000 \mathrm{pg} / \mathrm{mL}$ for rasagiline. The lower limit of quantification was $20.0 \mathrm{pg} / \mathrm{ml}$. The precision range of each concentration quality control sample (\% CV) was 3.5\%-6.5\%. The mean bias (\%) from the theoretical concentration of QC samples was between- $0.5 \%$ and $3.3 \%$ for rasagiline.

PK and BE Analysis

PK parameters such as $\mathrm{AUC}_{0-\mathrm{t}}, \mathrm{AUC}_{0-[?]}, \mathrm{C}_{\max }$, the elimination half-life $\left(\mathrm{T}_{1 / 2}\right)$, and time to achieve the $\mathrm{C}_{\max }\left(\mathrm{T}_{\max }\right)$ were determined. The pharmacokinetic parameters of rasagiline were calculated with WinNonlin Version 6.4 by non-compartment model. The AUC was calculated by the linear trapezoidal linear interpolation method. The experimental results were mainly analyzed by descriptive statistics using SAS (SAS Institute, USA, version 9.4). The main pharmacokinetic indexes after logarithmic transformation were analyzed by a mixed effect model. The level of significance was set at $\mathrm{P}<0.05$.

\section{Results}

Subjects Demographic characteristics

In the fasting group, a total of 147 subjects were screened and assessed for eligibility and 36 of them were enrolled. In the postprandial group, a total of 207 subjects were screened and 72 subjects were enrolled. During the whole study, only 1 participant withdrew due to an adverse event of tachycardia after one administration. The flow chart of subjects distribution is shown in Figure 1. The demographic characteristics for each group are shown in Table 1.

\section{A Postprandial group}

\section{B Fasting group}

Figure 1 Study subjects disposition flow diagram

\section{Table 1. Population Data}

\begin{tabular}{lll}
\hline Parameters & Fasting & Postprandial \\
$\mathrm{N}$ & 36 & 72 \\
Gender n (\%) Male Female & $20(55.6 \%) 16(44.4 \%)$ & $62(86.1 \%) 10(13.9 \%)$ \\
Age (y) Mean \pm SD Min-Max & $30.4 \pm 7.5518-45$ & $30.7 \pm 6.6418-45$ \\
Weight (kg) Mean \pm SD Min-Max & $66.12 \pm 8.85446 .6-88.4$ & $65.44 \pm 9.21647 .6-93.1$ \\
BMI (kg/m2) Mean \pm SD Min-Max & $24.33 \pm 2.22319 .8-27.3$ & $23.15 \pm 2.269$ 19.1-27.8 \\
\hline
\end{tabular}

BMI indicates body mass index;Max,maximum;Min,minimum.

Pharmacokinetic calculations

Under fasted and postprandial states, rasagiline $\mathrm{PK}$, measured using the geometric mean $\mathrm{AUC}_{0-\mathrm{t}}, \mathrm{AUC}_{0-[?]}$ , and $\mathrm{C}_{\max }$, were similar between test drug and reference drug (Table 2 and Table 3). The mean rasagiline plasma concentration-time profiles stratified by treatment are depicted in Figure 2 and Figure 3. Median $\mathrm{T}_{\max }$ was similar between the test drug and reference drug. The detailed pharmacokinetic parameters of the two rasagiline tablets using single dosing under fasted and postprandial conditions are presented in Table 2 and Table 3. 


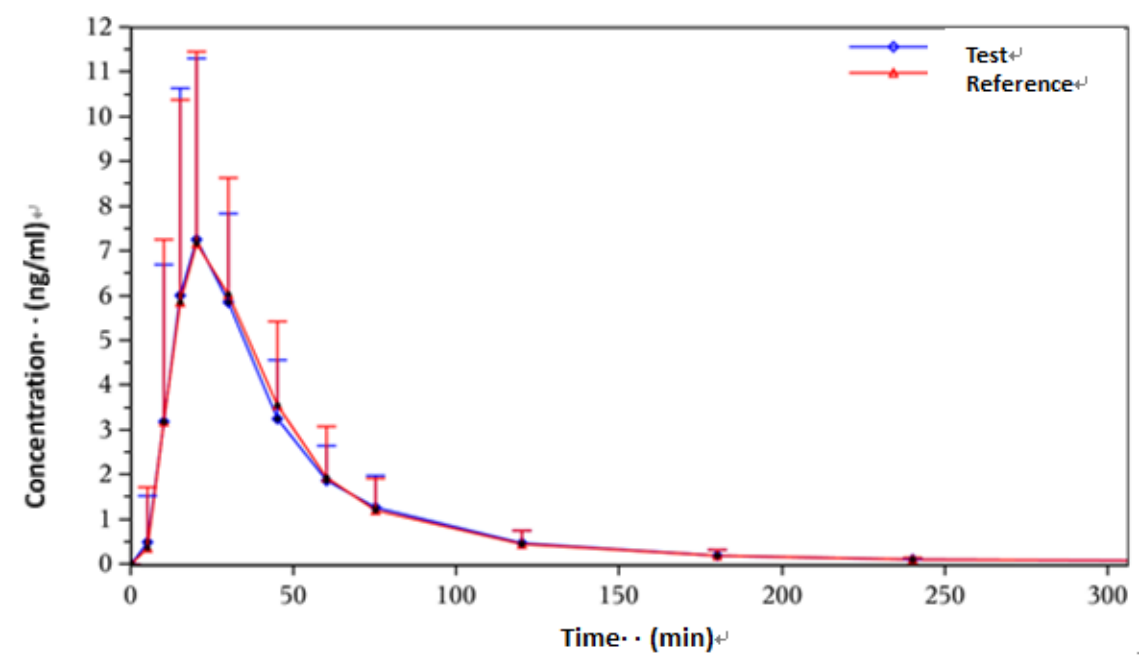

Figure 2. Concentration-time Curves Under Fasting Conditions.

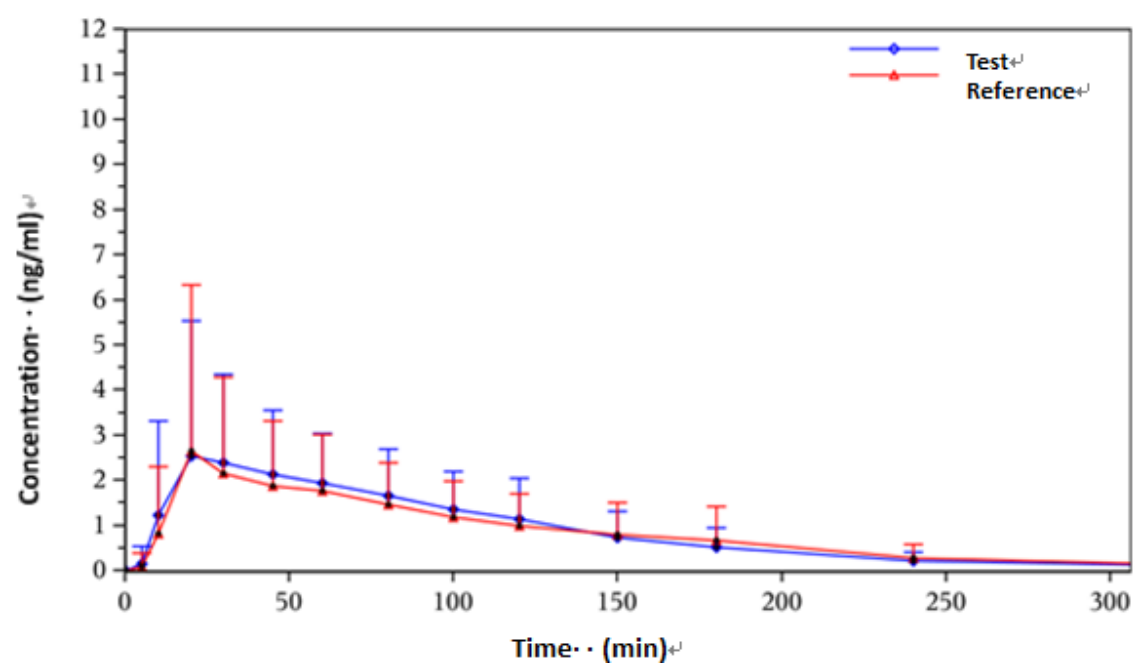

Figure 3. Concentration-time Curves Under Postprandial Conditions.

Table 2. Pharmacokinetic Parameters Under Fasting Conditions

\begin{tabular}{lll}
\hline Parameters & Mean $\pm \mathrm{SD}(\mathrm{CV} \%)$ & Mean $\pm \mathrm{SD}(\mathrm{CV} \%)$ \\
& Test $(\mathrm{N}=18)$ & Reference $(\mathrm{N}=18)$ \\
$\mathrm{T}_{\max }(\mathrm{h})$ & $0.33(0.17,0.75)(30.96)$ & $0.33(0.17,0.75)(36.83)$ \\
$\mathrm{C}_{\max }(\mathrm{ng} / \mathrm{mL})$ & $8.53 \pm 3.60(42.16)$ & $8.87 \pm 4.13(46.52)$ \\
$\mathrm{AUC}_{0-\mathrm{t}}\left(\mathrm{h}^{*} \mathrm{ng} / \mathrm{ml}\right)$ & $5.84 \pm 1.66(28.44)$ & $5.91 \pm 1.80(30.40)$ \\
$\mathrm{AUC}_{0-[?]}\left(\mathrm{h}^{*} \mathrm{ng} / \mathrm{ml}\right)$ & $5.99 \pm 1.72(28.65)$ & $6.10 \pm 1.90(31.04)$ \\
$\mathrm{t}_{\frac{1}{2}}(\mathrm{~h})$ & $3.03 \pm 1.51(50.06)$ & $3.49 \pm 2.45(70.20)$ \\
\hline
\end{tabular}

$\mathrm{AUC}_{0-\mathrm{t}}$ indicates area under the concentration-time curve from 0 to t; $\mathrm{AUC}_{0-[?]}$, area under the concentrationtime curve from 0 to infinity; $\mathrm{C}_{\max }$, maximum concentration; $\mathrm{CV}$, coefficient of variation; $\mathrm{t}_{1 / 2}$, half-life of 
elimination; $\mathrm{T} \max$, time to $\mathrm{C}_{\max }$.

$\mathrm{T}_{\max }$ was represented as median (min, max) (CV\%).

Table 3. Pharmacokinetic Parameters Under Postprandial Conditions

\begin{tabular}{lll}
\hline Parameters & Mean $\pm \mathrm{SD}(\mathrm{CV} \%)$ & Mean $\pm \mathrm{SD}(\mathrm{CV} \%)$ \\
& Test $(\mathrm{N}=18)$ & Reference $(\mathrm{N}=18)$ \\
$\mathrm{T}_{\max }(\mathrm{h})$ & $0.75(0.17,3.00)(76.15)$ & $1.00(0.17,3.00)(77.57)$ \\
$\mathrm{C}_{\max }(\mathrm{ng} / \mathrm{mL})$ & $4.33 \pm 2.43(56.18)$ & $4.36 \pm 2.98(68.36)$ \\
$\mathrm{AUC}_{0-\mathrm{t}}\left(\mathrm{h}^{*} \mathrm{ng} / \mathrm{ml}\right)$ & $4.93 \pm 1.36(27.60)$ & $4.81 \pm 1.38(28.75)$ \\
$\mathrm{AUC}_{0-[?]}\left(\mathrm{h}^{*} \mathrm{ng} / \mathrm{ml}\right)$ & $5.01 \pm 1.39(26.65)$ & $4.89 \pm 1.40(28.67)$ \\
$\mathrm{t}_{\frac{1}{2}}(\mathrm{~h})$ & $1.82 \pm 0.99(54.37)$ & $1.97 \pm 3.02(153.34)$ \\
\hline
\end{tabular}

$\mathrm{AUC}_{0-\mathrm{t}}$ indicates area under the concentration-time curve from 0 to $\mathrm{t} ; \mathrm{AUC}_{0-[?]}$, area under the concentrationtime curve from 0 to infinity; $\mathrm{C}_{\max }$, maximum concentration; $\mathrm{CV}$, coefficient of variation; $\mathrm{t}_{1 / 2}$, half-life of elimination; $\mathrm{T} \max$, time to $\mathrm{C}_{\max }$.

$\mathrm{T}_{\max }$ was represented as median $(\min , \max )(\mathrm{CV} \%)$.

Bioequivalence

Except for one subject who withdrew after the first cycle, a total of 71 subjects entered the BE assessment. The geometric mean ratios (GMRs) $(90 \% \mathrm{CI})$ of $\mathrm{C}_{\max }, \mathrm{AUC}_{0-\mathrm{t}}$ and $\mathrm{AUC}_{0-[?]}$ were $97.84 \%, 99.60 \%$ and $99.12 \%$ under fasting condition, and the GMRs $(90 \% \mathrm{CI})$ of $\mathrm{C}_{\max }, \mathrm{AUC}_{0-\mathrm{t}}$ and $\mathrm{AUC}_{0-[?]}$ were $102.89 \%, 103.41 \%$ and $103.25 \%$ under postprandial condition. All these $90 \%$ CIs were within the range of BE from FDA guidelines ( $80 \%$ to 125\%). The results are shown in Table 4 and Table 5. A sensitivity analysis in Table 6 was performed to evaluate the influence of the dropout including data from the withdraw subject who completed the first cycle only. The GMRs (90\%CI) of $\mathrm{C}_{\max }, \mathrm{AUC}_{0-\mathrm{t}}$ and $\mathrm{AUC}_{0-[?]}$ were $102.86 \% 103.39 \%$ and $103.22 \%$. These $90 \%$ CIs from sensitivity analysis were within the same range of $\mathrm{BE}$.

Table 4. Bioequivalence Under Fasting Conditions

\begin{tabular}{|c|c|c|c|c|c|}
\hline PK parameter & $\begin{array}{l}\text { GM and } \mathrm{GMR}, \mathrm{N}=36 \\
\mathrm{~T}\end{array}$ & $\begin{array}{l}\text { GM and } \mathrm{GMR}, \mathrm{N}=36 \\
\mathrm{R}\end{array}$ & $\begin{array}{l}\text { GM and GMR,N=36 } \\
\text { GMR }(\%)\end{array}$ & $\mathrm{CV} \%$ & $90 \% \mathrm{CI}$ \\
\hline $\mathrm{C}_{\max }(\mathrm{ng} / \mathrm{ml})$ & 7.82 & 7.99 & 97.84 & 26.30 & 88.26108 .46 \\
\hline $\mathrm{AUC}_{0-\mathrm{t}}\left(\mathrm{h}^{*} \mathrm{ng} / \mathrm{ml}\right)$ & 5.60 & 5.62 & 99.60 & 14.15 & 94.16105 .35 \\
\hline $\mathrm{AUC}_{0-[?]}\left(\mathrm{h}^{*} \mathrm{ng} / \mathrm{ml}\right)$ & 5.74 & 5.79 & 99.12 & 14.58 & 93.55105 .01 \\
\hline
\end{tabular}

GMR refers to the geometric mean ratio of the test over reference pharmacokinetic metric.

Table 5. Bioequivalence Under Postprandial Conditions

\begin{tabular}{|c|c|c|c|c|c|}
\hline PK parameter & $\begin{array}{l}\text { GM and } \mathrm{GMR}, \mathrm{N}=72 \\
\mathrm{~T}\end{array}$ & $\begin{array}{l}\text { GM and } \mathrm{GMR}, \mathrm{N}=72 \\
\mathrm{R}\end{array}$ & $\begin{array}{l}\text { GM and GMR,N=72 } \\
\operatorname{GMR}(\%)\end{array}$ & CV\% & $90 \% \mathrm{CI}$ \\
\hline $\mathrm{C}_{\max }(\mathrm{ng} / \mathrm{ml})$ & 3.76 & 3.66 & 102.89 & 52.89 & 89.54118 .23 \\
\hline $\mathrm{AUC}_{0-\mathrm{t}}\left(\mathrm{h}^{*} \mathrm{ng} / \mathrm{ml}\right)$ & 4.76 & 4.61 & 103.41 & 12.45 & 99.88107 .07 \\
\hline $\mathrm{AUC}_{0-[?]}\left(\mathrm{h}^{*} \mathrm{ng} / \mathrm{ml}\right)$ & 4.84 & 4.70 & 103.25 & 12.94 & 99.59107 .05 \\
\hline
\end{tabular}

GMR refers to the geometric mean ratio of the test over reference pharmacokinetic metric.

Table 6. Sensitivity Analysis Under Postprandial Conditions 


\begin{tabular}{|c|c|c|c|c|c|}
\hline PK parameter & $\begin{array}{l}\text { GM and } \mathrm{GMR}, \mathrm{N}=72 \\
\mathrm{~T}\end{array}$ & $\begin{array}{l}\text { GM and } \mathrm{GMR}, \mathrm{N}=72 \\
\mathrm{R}\end{array}$ & $\begin{array}{l}\text { GM and GMR,N=72 } \\
\text { GMR (\%) }\end{array}$ & CV\% & $90 \% \mathrm{CI}$ \\
\hline $\mathrm{C}_{\max }(\mathrm{ng} / \mathrm{ml})$ & 3.76 & 3.66 & 102.89 & 52.89 & 89.59118 .11 \\
\hline $\mathrm{AUC}_{0-\mathrm{t}}\left(\mathrm{h}^{*} \mathrm{ng} / \mathrm{ml}\right)$ & 4.76 & 4.61 & 103.41 & 12.45 & 99.86107 .03 \\
\hline $\mathrm{AUC}_{0-[?]}\left(\mathrm{h}^{*} \mathrm{ng} / \mathrm{ml}\right)$ & 4.84 & 4.70 & 103.25 & 12.94 & 99.57107 .01 \\
\hline
\end{tabular}

Safety and Tolerability

Data from 108 subjects were included in this safety evaluation. Of the 36 subjects enrolled in the fasting group, all completed the two cycle study. Of these, 10 subjects (27.8\% of 36) reported 17 drug-related AEs. Of the $17 \mathrm{AEs}, 6$ subjects (16.7\% of 36) experienced 6 post-dose AEs related to the test product and 6 subjects (16.7\% of 36) experienced 11 post-dose AEs related to the reference product. In terms of the severity of AEs, all AEs were classified as level 1 to level 2 (NCI CTCAE 4.03), and there were no AEs of level 3 or above. No severe AEs (SAE) occurred during the treatment.

In the postprandialgroup, 72 subjects were recruited and 71 subjects completed both periods of the study. A total of 19 subjects (26.4\% of 72 ) reported 37 drug-related AEs. 10 subjects (13.9\% of 72 ) experienced 14 post-dose AEs related to the test product and 10 subjects (13.9\% of 72) experienced 23 post-dose AEs related to the reference product. Among these AES, there were two events of hypertension, severity level 3, related to $\mathrm{T}$ and $\mathrm{R}$ tablet administrations respectively. In this group, one subject withdrew due to tachycardia. No SAEs or deaths were observed during the study.

During the last scheduled visit, all AEs were resolved in both groups.

\section{Discussion}

In addition to a BE study under fasting conditions, the US. FDA and National Medical Products Administration (NMPA) currently recommends a BE study under fed conditions should be conducted for all orally administered drug products submitted as an ANDA, with only a few specific exceptions: when the drug substance is considered a BCS class 1 drug, and dissolution, solubility, and permeability data support a biowaiver of in vivo BE testing, or When the DOSAGE AND ADMINISTRATION section of the reference listed drug (RLD) label states that the product should be taken only on an empty stomach, or When the RLD label does not make any statements about the effect of food on absorption or administration [15-16]. Rasagiline is a highly solube low permeable drug and therefore classifies as a BCS class III product. According to the drug label of RLD, rasagiline can be administered with or without food, because AUC is not signifcantly affected by food ${ }^{[9]}$. Therefore, this BE study for rasagiline was conducted under both fasting and postprandial conditions. The main PK parameters ( $\mathrm{T}_{\max }$ and $\mathrm{AUC}$ ) of the test drug and the reference rasagiline had similar performances under both fasting and postprandial conditions. The $90 \%$ CIs of the GMRs for $\mathrm{AUC}$ and $\mathrm{C}_{\max }$ of the two tablets were within the $\mathrm{BE}$ range.

As shown above, rasagiline was rapidly absorbed by the gastrointestinal tract, with $\mathrm{T}_{\max }$ occurring between 0.17 and 0.75 hours after a single $1 \mathrm{mg}$ dose under fasting conditions. After a high-fat meal, $\mathrm{T}_{\max }$ of rasagiline is delayed from 0.33 hours under fasting conditions to 1 hour under postprandial conditions. This shows some difference from previous studies which demonstrated that food did not affect $\mathrm{T}_{\max }$ of rasagiline, although $\mathrm{C}_{\max }$ and exposure (AUC) were decreased by approximately $60 \%$ and $20 \%$, respectively, when the drug was taken with a high-fat meal ${ }^{[8-9]}$. In our study, we also found that while a high-fat meal has significant effects on the absorption rate of rasagiline,but minor effects on the absorption amount. Compared with fasting conditions, the $\mathrm{C}_{\max }$ of rasagiline measured after high-fat meal decreased significantly $(4.36 \pm 2.98$ vs $8.87 \pm 4.13$ ), and the AUC decreased slightly (4.89 \pm 1.40 vs $6.10 \pm 1.90)$, which was consistent with prior literature.

Study shows that in most cases, increased bioavailability with food resulted in decrease of intra-individual variation (IIV) of AUC. While food has a negative effect on a drug's bioavailability, administering the drug 
with food resulted in greater IIV compared to administering it without food ${ }^{[17]}$. In our study we found that, when administered with a high-fat meal, corresponding to the decrease of $\mathrm{C}_{\max }$ is a significant increase in CV (26.3\% under fasting conditions vs $52.89 \%$ under postprandial conditions).

As stated above, administration with food can influence the absorption and systemic exposure of rasagiline. The effect of food on oral bioavailability results from a complex interplay of drug, formulation, food components, gastric and intestinal physiology (e.g. gastrointestinal pH, gastric emptying, intestinal transit ${ }^{[15,18,19]}$. The difference in PK parameters obtained may have resulted from differences in subjects, sample sizes, sample detectors, or other unknown factors. Additional considerations such as interindividual variability may also have contribution to this difference ${ }^{[11]}$.

Results from previous studies suggested that rasagiline was a highly variable drug (HVD), which was consistent with our study ${ }^{[17,20]}$. In 2016, a randomized, four-period, two-sequence, single-dose, replicate-crossover bioequivalence study of rasagiline was conducted in 30 healthy, adult male volunteers under fasting conditions by Van Rijswick YGJ. In Van Rijswick YGJ's studies, the IIV for $\mathrm{C}_{\max }$ of the reference product was $34.71 \%{ }^{[20]}$. In our study after co-administration with food, rasagiline IIV for $\mathrm{C}_{\max }$ was up to $52.89 \%$ under postprandial conditions, much higher than data from Van Rijswick YGJ's studies under fasting conditions. Traditionally statistical analysis of BE study data is performed by obtaining the average bioequivalence (ABE) approach via a two-way crossover design. Two products are deemed bioequivalent when the $90 \%$ CIs of the GMRs for AUC and $\mathrm{C}_{\max }$ fall within the limits of $80-125 \%$ by ABE. Establishing BE for HVD is challenging as high IIV requires a dramatically larger sample size. Since 2006, FDA has accepted a referencescaled average bioequivalence (RSABE) approach for HVDs. Using the RSABE approach, the BE study can use either a partial replicate (three-way crossover, RTR, RRT, or TRR) or full replicate (four-way crossover, RTRT or TRTR) design, and the BE limits are broadened to greater than $80-125 \%{ }^{[21-24]}$. This BE study uses a two-way crossover design, meeting the ABE criteria which was also required by the National Medi-

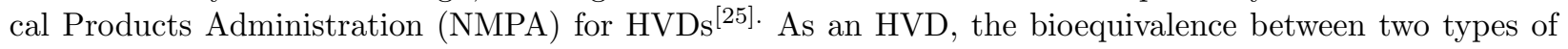
rasagiline was established by expanding the sample size of the postprandial group to 72 cases.

In addition to PK bioequivalence, both types of rasagiline were well tolerated with no significant differences between safety profiles. The occurrence and severity of AEs in our study was similar to results from previous studies and described under the the rasagiline label.

\section{Conclusions}

Data from this study show that there is no difference in absorption rate and effect between the two preparations of rasagiline. All subjects showed good tolerance of both preparations without any serious or unexpected adverse reactions. According to the relevant provisions of NMPA guidelines, the two tablets were considered bioequivalent for single dosing under fasting and postprandial conditions.

\section{Acknowledgments}

This work was supported by Changzhou Siyao Pharmaceuticals Co.,Ltd.

\section{Conflicts of Interest}

None of the authors have any conflicts of interest to report.

\section{Author Contributions}

All authors critically reviewed the manuscript and contributed to conception and design of the protocol, data analysis, and interpretation. Yinjuan Li and Xinghe Wang had involved in drafting the manuscript or revising it critically for important intellectual content.

\section{References}

1. M.T. Hayes. Parkinson's disease and Parkinsonism. Am. J. Med. 2019;132:802-807. 
2. P.A. Lewis, J.E. Spillane, Chapter 3 Parkinson's disease, in: P.A. Lewis, J.E. Spillane (Eds.). The molecular and clinical pathology of neurodegenerative disease. Academic Press; 2019. pp. 83-121.

3. C. Raza, R. Anjum, N.U.A. Shakeel. Parkinson's disease: mechanisms, translational models and management strategies. Life Sci. 2019; $226: 77-90$.

4. Zou YM, Liu J, Tian ZY, Lu D, Zhou YY. Systematic review of the prevalence and incidence of Parkinson's disease in the People's Republic of China. Neuropsychiatr Dis Treat. 2015;11:1467-72.

5. Finberg JPM. The discovery and development of rasagiline as a new anti-Parkinson medication. J Neural Transm (Vienna). 2020 Feb;127(2):125-130.

6. Avila A, Caballol N, Martín-Baranera M, Gómez-Ruiz I, Balagué-Marmaña M, Planas-Ballvé A, Cardona $\mathrm{X}$. Rasagiline and safinamide as a dopamine-sparing therapy for Parkinson's disease. Acta Neurol Scand. 2019 Jul;140(1):23-31.

7. Thébault JJ, Guillaume M, Levy R. Tolerability, safety, pharmacodynamics, and pharmacokinetics of rasagiline: a potent, selective, and irreversible monoamine oxidase type B inhibitor. Pharmacotherapy. 2004 Oct;24(10):1295-305.

8. Chen JJ, Swope DM. Clinical pharmacology of rasagiline: a novel, second-generation propargylamine for the treatment of Parkinson disease. J Clin Pharmacol. 2005 Aug;45(8):878-94.

9. Azilect prescribing information, 2018. Available at:

http://www.azilect.com/azilect.pdf.

10. Zhang Z, Wang J, Chen S, et al. Efficacy and safety of rasagiline in Chinese patients with early Parkinson's disease: a randomized, double-blind, parallel, placebo-controlled, fixed-dose study. Transl Neurodegener. 2018 Dec 6;7:32.

11. Elgart A, Rabinovich-Guilatt L, Eyal E, Gross A, Spiegelstein O. Pharmacokinetics and safety of single and multiple doses of rasagiline in healthy Japanese and caucasian subjects. Basic Clin Pharmacol Toxicol. 2019 Mar;124(3):273-284.

12. Hattori N, Takeda A, Takeda S, Nishimura A, Kato M, Mochizuki H, Nagai M, Takahashi R. Efficacy and safety of adjunctive rasagiline in Japanese Parkinson's disease patients with wearing-off phenomena: A phase 2/3, randomized, double-blind, placebo-controlled, multicenter study. Parkinsonism Relat Disord.2018 Aug; $53: 21-27$.

13. Chen, X., Zhao, Q., Jiang, J.et al. Pharmacokinetics of Rasagiline in Healthy Adult Chinese Volunteers with Various Genotypes: A Single-Center, Open-Label, Multiple-Dose Study. Clin Drug Investig. 2016;36:369-376.

14. Pintér, D., Lajtos, J., Janszky, J. and Kovács, N. Clinical Experience with Generic Rasagiline (Ralagoß) in Patients with Parkinson's Disease: An Open-Label, Multicenter, Observational Study. Advances in Parkinson's Disease. 2019; 8:18-34.

15.US Food and Drug Administration. Guidance for industry: food-effect bioavailability and fed bioequivalence studies, 2002. Available at:https://www.fda.gov/media/70945/download (accessed 22.04.2020).

16.National Medical Products Administration. Technical guidelines for the study of human bioequivalence in the pharmacokinetics of chemical generic drugs, 2016. Available at:http://www.nmpa.gov.cn/WS04/CL2138/300138.html. (accessed 22.04.2020).

17. Kang SP, Ratain MJ. Inconsistent labeling of food effect for oral agents across therapeutic areas: differences between oncology and non-oncology products. Clin Cancer Res. 2010 Sep 1;16(17):4446-51.

18.Fleisher D, Li C, Zhou Y, Pao L, Karim. A Drug, meal and formulation interactions influencing drug absorption after oral administration: clinical implications. ClinPharmacokinet. 1999;36:233-254 
19.Welling PG. Effects of food on drug absorption. Annu Rev Nutr. 1996;16:383-415.

20. Van Rijswick YGJ, Quesada MJ, Van Os SHG. Bioequivalence between generic rasagiline (As Tartrate) and rasagiline (As Mesylate). J Bioequiv. 2016;2(1):104.

21. US Food and Drug Administration. Guidance for industry bioavailability and bioequivalence studies submitted in NDAs or INDs-general considerations[EB /OL]2014. Available at:

https://www.fda.gov/media/88254/download. (accessed 22.04.2020).

22. US Food and Drug Administration. Draft guidance on progesterone, 2011. Available at:https://www.accessdata.fda.gov/dr docs/psg/Progesterone_caps_19781_RC02-11.pdf(accessed 22.04.2020).

23. European Medicine Agency. Guideline on the investigation of bioequivalence, 2010. Available at:https://www.ema.europa. guideline/guideline-investigation-bioequivalence-rev1_en.pdf(accessed 22.04.2020).

24.Davit B, Chen M-L, Conner DP et al. Implementation of a reference-scaled average bioequivalence approach for highly variable generic drug products by the US Food and Drug Admininstration. AAPS J . 2012;14:915-924.

25. National Medical Products Administration. Technical guidelines for bioequivalence study of highly variable drugs,2018. Available at:http://www.nmpa.gov.cn/WS04/CL2093/331454.html. (accessed 22.04.2020). 